\title{
Nanoscale Wear as a Stress-Assisted Chemical Reaction: An in-situ TEM Study
}

\author{
Robert W. Carpick ${ }^{1}$ and Tevis D.B. Jacobs ${ }^{2}$. \\ 1. Dept. of Mechanical Engineering \& Applied Mechanics, University of Pennsylvania, Philadelphia, PA, \\ USA \\ 2. Dept. of Materials Science \& Engineering, University of Pennsylvania, Philadelphia, PA, USA
}

Wear of sliding contacts leads to energy dissipation and device failure, with massive economic and environmental costs [1]. However, wear phenomena are typically described empirically, since physical and chemical interactions at sliding interfaces are not fully understood at any length-scale. Fundamental insights from individual nanoscale contacts are crucial for understanding wear at larger length-scales [2], and for enabling reliable nanoscale devices, manufacturing, and microscopy. Observed nanoscale wear mechanisms include fracture [3] and plastic deformation [4], but recent experiments [5-7] and simluations [8] propose another mechanism: wear via atom-by-atom removal ("atomic attrition") modeled using stress-assisted chemical reaction kinetics. Experimental evidence for this has so far been inferential.

Here we quantitatively measure wear of silicon, a material relevant to small-scale devices, using a nanoindentor (Hysitron, Inc.) operated in situ inside a transmission electron microscopy (TEM) [9] (Figure 1). We resolve worn volumes as small as $25 \pm 5 \mathrm{~nm}^{3}$, a factor of $10^{3}$ lower than alternative techniques $[10,11]$. Wear of silicon against diamond is consistent with atomic attrition, and inconsistent with fracture or plastic deformation, which we explicitly rule out by direct imaging. The rate of atom removal depends exponentially on stress in the contact, as predicted by chemical rate kinetics [12]. Measured activation parameters are consistent with an atom-by-atom process [13]. These results establish atomic attrition as the primary wear mechanism of silicon at low loads through direct observation [14].

\section{References:}

[1] H.P. Jost, Wear 136, 1 (1990).

[2] C.M. Mate, Tribology on the small scale: A bottom up approach to friction, lubrication, and wear

(Oxford University Press, Oxford ; New York, 2008), pp. xiii.

[3] J. Liu, D.S. Grierson, J. Notbohm, S. Li, S.D. O'Connor, K.T. Turner, R.W. Carpick, P. Jaroenapibal, A.V. Sumant, J.A. Carlisle, N. Neelakantan, and N. Moldovan, Small 6, 1140 (2010).

[4] A.P. Merkle, and L.D. Marks, Wear 265, 1864 (2008).

[5] E. Gnecco, R. Bennewitz, and E. Meyer, Phys. Rev. Lett. 88, 215501/1 (2002).

[6] B. Gotsmann, and M.A. Lantz, Phys. Rev. Lett. 101, 125501 (4 pp.) (2008).

[7] H. Bhaskaran, A. Sebastian, and M. Despont, IEEE Transactions on Nanotechnology 8, 128 (2009).

[8] G.T. Gao, P.T. Mikulski, and J.A. Harrison, J. Am. Chem. Soc. 124, 7202 (2002).

[9] T.D. Jacobs, and R.W. Carpick, Nature Nanotech. 8, 108 (2013).

[10] J. Liu, J.K. Notbohm, R.W. Carpick, and K.T. Turner, ACS Nano 4, 3763 (2010).

[11] K.H. Chung, and D.E. Kim, Tribol. Lett. 15, 135 (2003).

[12] P. Hanggi, Talkner, P. \& Borkovec, M., Rev. Mod. Phys. 62, 251 (1990). 
[13] K.J. Van Vliet, L. Ju, Z. Ting, S. Yip, and S. Suresh, Phys. Rev. B 67, 104105 (2003).

[14] I.-. Use of the facilities of the Pennsylvania Regional Nanotechnology Facility is acknowledged. Funding from the National Science Foundation (Grants CMMI-0826076, and DGE-0221664) is gratefully acknowledged.

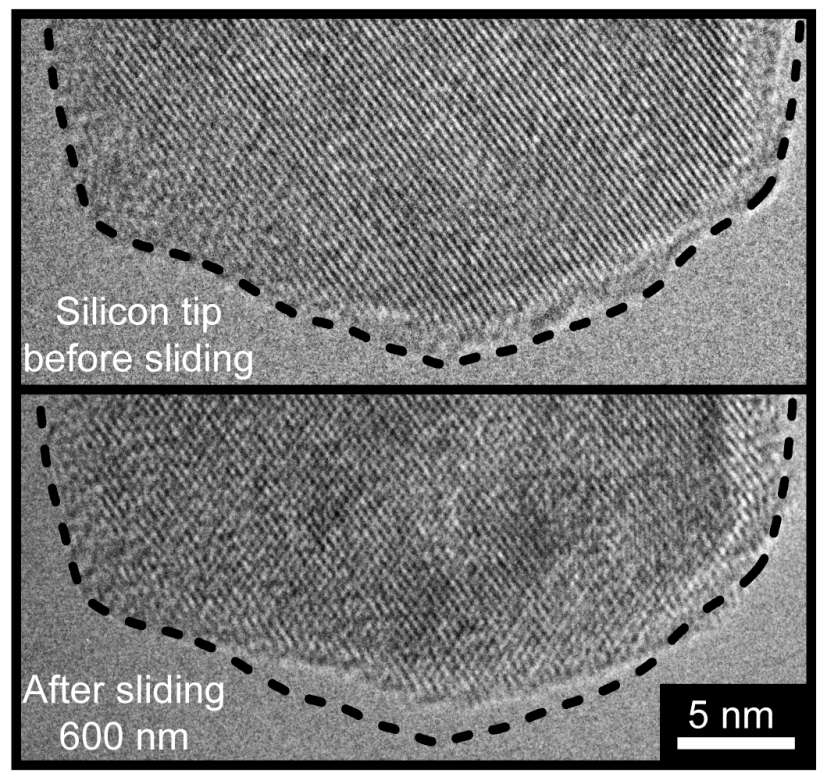

Figure 1. TEM image of a single crystal silicon AFM probe, before and after sliding $600 \mathrm{~nm}$ against a diamond surface in the TEM. In both images, the dotted line indicates the profile of the tip prior to sliding. Thus, the amount of material eroded during the experiment can be identified in the lower image. 